\title{
Three-dimensional Inspection and Characterization of Plastic Micro Mixers Fabricated by Different Packaging Techniques
}

\author{
R. Y. Zhang, K. Chen", L. X. Wang, W. J. Zhu \\ Department of Materials and Energy Engineering, Guongdong University of Technology, \\ Guongzhou 510090, China. \\ *Department of Mechanical Engineering, University of Utah, Salt Lake City, UT 84112, USA \\ (Ph.No.-01-801-5814150.E-mail: chen@mech.utah.edu)
}

\begin{abstract}
An X-ray microtomography system was used for inspecting and characterizing the packaging of micro mixers of planar configuration. Two types of micro mixers were machined from acrylic plate by means of precision milling, and four different packaging techniques were tested for mixer assembly: adhesive bonding, plasma bonding, screw-bolting, and xurography with doublesided tape. The interiors of the mixers were thoroughly examined by navigating the threedimensional images generated by the micro CT system. It was found that plasma bonding did not work well on acrylics. The boundaries between the acrylic plate and the plastic tape used in tape bonding were almost indistinguishable in the X-ray images because the densities of these two materials are very close. Of the different packaging techniques we tested tape bonding provided good sealing without the drawback of glue or sealing materials smeared into the mixing chamber or flow channels.
\end{abstract}

Key Words : Characterization of plastic packaging, Micro-CT (Computed Tomography), Micro mixer

\section{INTRODUCTION}

X-ray based technologies have been commonly used for the inspection and characterization of a variety of natural and man-made materials and devices. For instance, Stojanovic used the X-ray powder diffraction technique to determine the structural and microstructural parameters of Bdoped $\mathrm{PbTe}$ semiconductor [1]. X-ray diffraction patterns of iron oxide powders were analyzed 
in Shi and Hwang's investigation of microwave-assisted miniaturization of chemical plants [2]. $\mathrm{X}$-ray micro CT (Computed Tomography) is a very popular technique for nondestructive inspection and characterization of structures, materials, as well as microelectronics and MEMS devices. Nano-focus X-ray sources are commercially available in recent years, and highresolution Nano-CT has been developed [3] for materials research [4].

Micro mixers of planar configuration are the main components of today's micro sensors and micro biological, medical, and chemical reactors/analyzers, which can be batch-fabricated and easily combined with microelectronics using the integrated circuit technology. Mixing of two or more fluid streams is also encountered in other MEMS or microfluidic devices such as micro pumps and valves and micro heat engines. Plastics are the favorable materials for micro mixers. They cost less and are easier to handle and use than most other materials used in the fabrication of microfluidic devices and components. Most plastics are transparent, thus flow visualization is easy. This feature is important to medical devices for which easy and continuous monitoring of fluid flows are often required. A transparent shell also makes it convenient to inspect the mixer interiors, detect leakages, and study the flow inside the mixer.

Different plastic materials have been tested and a variety of packaging and assembly techniques developed for the fabrication of plastic micro mixers. At present adhesive bonding and hot embossing are the popular packaging techniques for plastic micro mixers. Innovative designs and methods (e.g., [5-9]) have been developed to improve bonding strengths and conditions, prevent adhesives and sealing materials smeared into the mixing chamber or flow channels, facilitate the packaging process, and reduce deformation during packaging. Plasma bonding is another technique that has been applied to the packaging of plastic microchips and microfluidics fabricated from plastics as well as glass and metals. Double-sided tapes were used to bond two glass slides in Greer et al's manufacturing of microfluidic channels [5].

The machined or etched surfaces in plastic mixers are often opaque, prohibiting visual inspection with a magnifier or optical microscope. Of the 3 nondestructive methods (IR transmission imaging, X-ray tomography, and acoustic microscopy) currently used to inspect bonded devices [10], X-ray imaging has gained increasing popularity for measuring and characterizing internal features and flaws of MEMS and microfluidics. However detailed information (e.g., [11]) about the application of X-ray CT for the inspection and characterization of microfluidics is rare. This motivated the present investigation of 3D (three-dimensional) X-ray images of micro mixers fabricated by different packaging techniques.

\section{FABRICATION AND PACKAGING OF MICRO MIXERS}

Two types of micro mixers of planar configuration were fabricated in the present investigation: a T-shaped mixer and a swirling-flow mixer that involves a circular mixing chamber with two tangential inlets and an axially directed outlet. Photos of these two micro mixers are given in 
figures 1 and 2. The pink powder in the photos is the dried red ink (Rhodamine) used in our flow visualization experiment. The rectangular mixing chamber of the T- mixer was fabricated out of a thin acrylic sheet and glued to another acrylic sheet to enable the mixing process to be observed easily. Two inlet and one exit channels were drilled into the mixing chamber after the two acrylic plates had been glued together. The diameter of the flow channels was approximately 0.4 $\mathrm{mm}$. The mixing chamber was approximately $20 \mathrm{~mm}$ long and $4 \mathrm{~mm}$ wide, and its thickness was $0.4 \mathrm{~mm}$ before packaging.
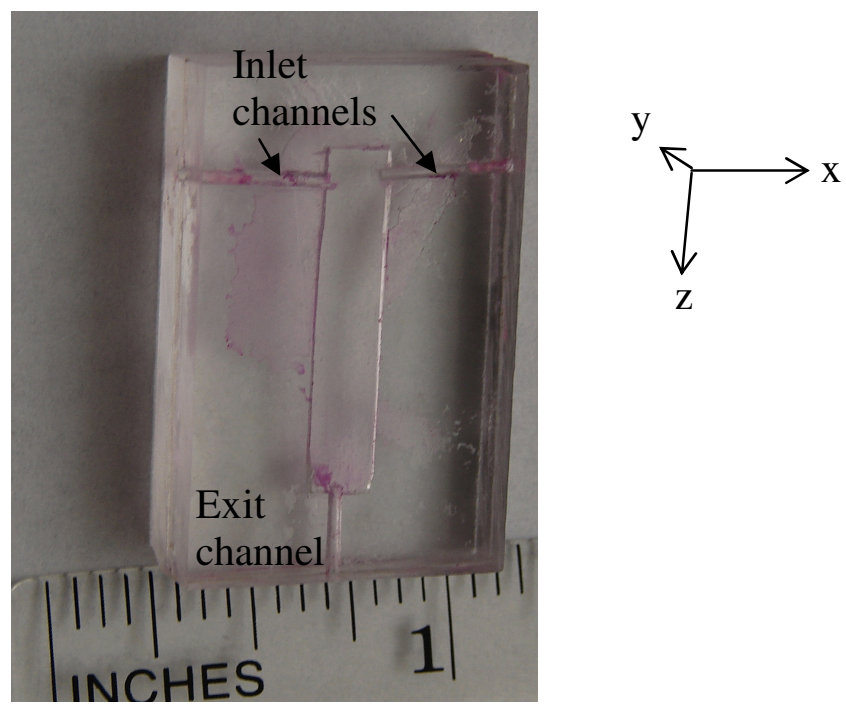

Figure 1. A T-shaped micro mixer fabricated from thin acrylic sheets.
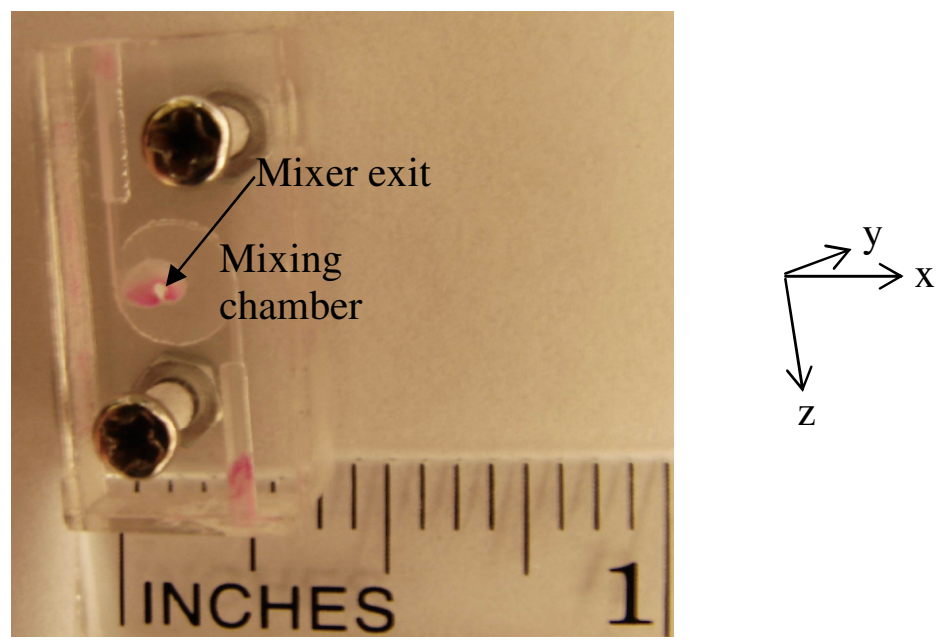

Figure 2. A swirling-flow micro mixer with two tangential inlets and an axially directed exit channel. 
The swirling-flow mixer was also fabricated from acrylic sheets for easy observation of the mixing process. The circular mixing chamber and two inlet channels were micro machined on one acrylic plate. The exit flow channel was drilled through another acrylic plate. The mixing chamber was approximately $0.4 \mathrm{~mm}$ deep before assembly and its diameter was $5 \mathrm{~mm}$. The two rectangular inlet channels were $1 \mathrm{~mm}$ wide and $1.5 \mathrm{~mm}$ deep at the mixing chamber rim. At about $2 \mathrm{~mm}$ upstream of the inlet ports the dimensions of the inlet channels increased to $2 \mathrm{~mm}$ by $2 \mathrm{~mm}$ for reduced frictional loss. The diameter of the exit channel was approximately $0.4 \mathrm{~mm}$. The surfaces of the mixing chambers of the 2 mixers were inspected using a SEM before packaging, and the roughness of the surfaces was found to be in the micron range.

Three different techniques were tested for the assembly of the swirling-flow mixer. The first method was to bolt the two acrylic plates together with screws. Because the surfaces of industrial acrylic sheets are extremely smooth, the two acrylic plates pressed against each other by the screws would make intimate contact. No bonding or sealing was applied to the contact surfaces of the two acrylic plates.

Plasma bonding was the second technique we tried to bond the two halves of the swirling-flow mixer. While this technique has been applied successfully to the bonding of a variety of materials, it failed to yield a good bonding between acrylic plates. Thus plasma bonding is probably not applicable to the packaging of MEMS or microfluidics fabricated from acrylics.

In the third technique a knife plotter by Graphtec cut the geometry of the contact surface out of $25 \mu \mathrm{m}$ thick, double coated tape by 3M. The tape was then sandwiched between the two acrylic plates before the plates were tightly bolted with two screws. In the last stage the tape-bonded acrylic mixer was placed in a $65^{\circ} \mathrm{C}$ oven for 45 minutes to ensure good bonding between the tape and the acrylic plates. Detailed information about this micro fabrication method can be found in Greer et al's paper [5].

\section{FACILITY FOR 3D NONDESTRUCTIVE INSPECTION}

The instrument used in the present investigation of micro mixer packaging is a custom-designed, X-ray micro-CT machine, Konoscope 40-130, by ARACOR. A microfocus X-ray tube by Kevex (Model number KM 13006) was used for X-ray generation with a focus spot of $8 \mu \mathrm{m}$ at 8 Watts, and $20 \mu \mathrm{m}$ at 16 Watts. The 2D (two-dimensional) cone-beam projections of this micro CT system have 2048 x 2048 pixels over a 10-mm diameter. The system magnification can be adjusted by changing the location of the specimen on a computer-controlled vertical/rotary positioning stage, allowing for the imaging of objects up to $40 \mathrm{~mm}$ in diameter. The maximum material density this micro CT system can handle is about $8 \times 10^{6} \mathrm{~kg} / \mathrm{m}^{3}$. 
The major component of the image detection system is a Photometrics XR300 camera with Thompson $2048 \times 2048$, Grade 1 CCD. It provides 16-bit digitization at $2 \times 10^{5}$ pixels per second. A Mac Power PC with LabView and LVCam installed was used to control the movements of the positioning stage and to acquire the CCD camera images. Three-dimensional tomographic images were reconstructed using an SGI Octane workstation. The software MIPAV (version 4) was used to display and navigate the 3D images. Detailed specifications and description of this custom-designed micro CT system can be found in Lin and Miller's paper [12].

\section{RESULTS AND DISCUSSION}

The 3D images re-constructed by the micro CT system allow a nondestructive inspection of the interiors of a micro mixer after the mixer has been packaged. The software MIPAV which was originally developed for medical research makes it convenient to view the scanned images at different locations from different angles, or to navigate the 3D images for animation making. Presented in figures 3 to 5 are the orthographic views of the X-ray images of the micro mixers we fabricated. The orientations of the Cartesian coordinates in these figures are shown in figures 1 and 2. Complete 3D data can be furnished upon request.

The three orthographic views of the T-mixer images at two different locations are shown in figures 3(a) and 3(b). The three panels from left to right in each figure are the $X$-ray images on the $\mathrm{X}-\mathrm{Y}, \mathrm{Y}-\mathrm{Z}$, and $\mathrm{X}-\mathrm{Z}$ planes. The origins of the local coordinate systems in these two figures are approximately at the middle points of the height (along the $\mathrm{Y}$ axis) and width (along the $\mathrm{X}$ axis) of the mixing chamber. The X-axis passes through the two inlet ports in figure 3(a), and is slightly upstream of the inlet ports in figure 3(b). The crosshairs in these images are the projections of the orthographic views in the other two directions. Each pixel in these images is about $20 \mu \mathrm{m}$. The dark areas in the images are voids (air), and the white areas are the adhesive. The gray areas represent the acrylics. Due to the large difference in densities of these three materials (air, adhesive, and acrylics), the boundaries between different materials are clearly distinguishable in the X-ray images.

At the locations marked by the crosshairs in figures 3(a) and 3(b) the mixer was well sealed by the adhesive. The white areas (the adhesive) in the central and right panels in figure 3 are in good alignment with the mixer contour except for the lower edges of the two inlet ports in the right panels. On the other hand the bonding was not very good as evidenced by the gaps in dark color (voids) between the surfaces of the acrylic plate and the adhesive in the two central panels. The finger-shape white areas attached to inlet ports in the right panels indicate some adhesive was smeared into the mixing chamber during packaging, and partially blocked the flow passageway in the neighborhood of the inlet ports. The left panel in figure 3(a) showed one of the inlet channels was also partially blocked by the adhesive. In the left panel in figure 3(b) the white 
area on the left has a slanted upper boundary, and the mixing chamber at this location is slightly narrower due to the smearing of the adhesive into the chamber.
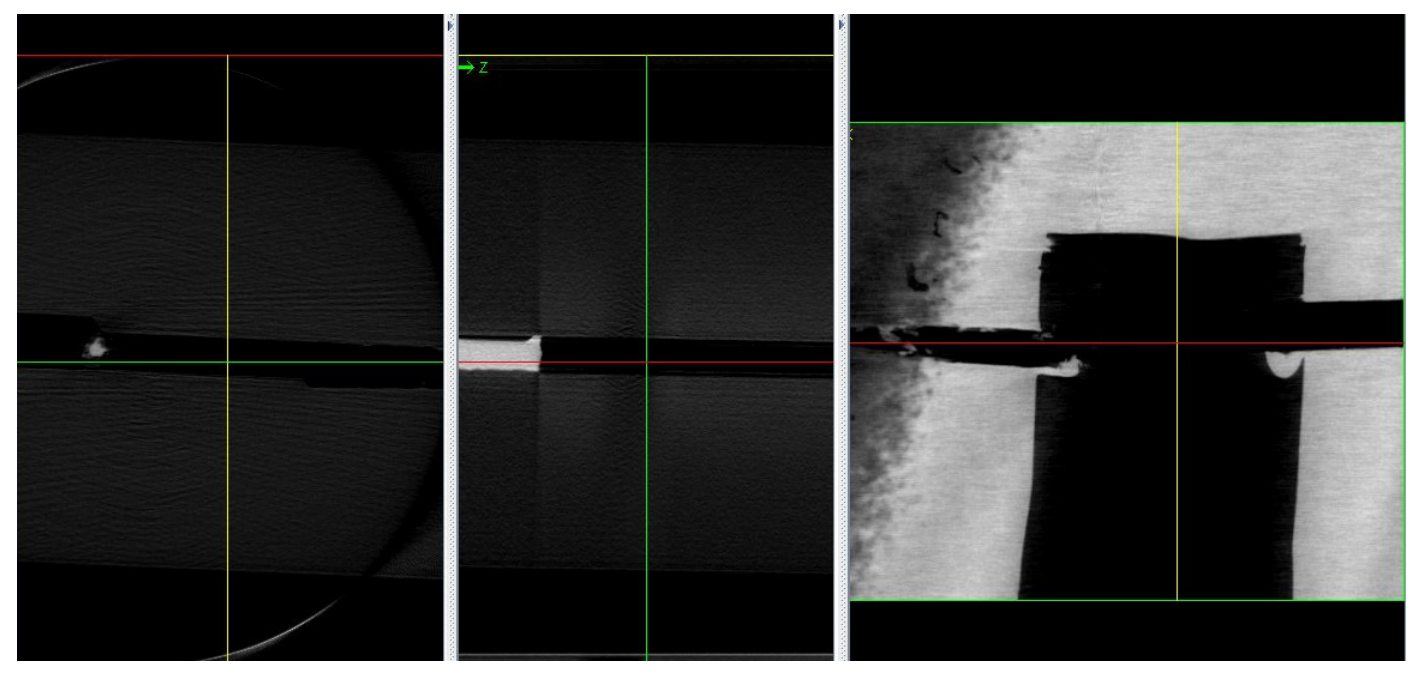

(a)
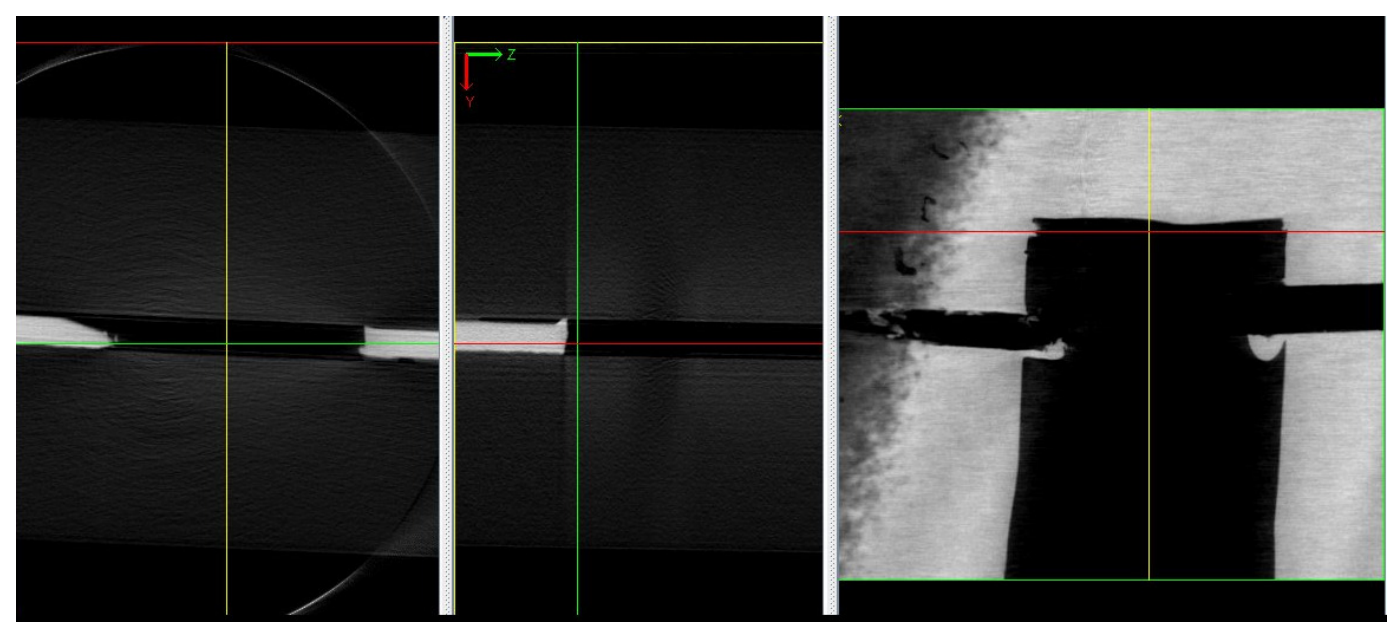

(b)
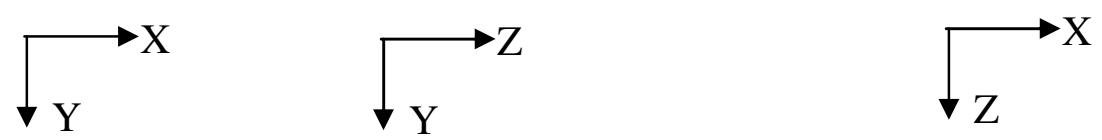

Figure 3. Orthographic views of the X-ray images at two different locations in the T-mixer. 

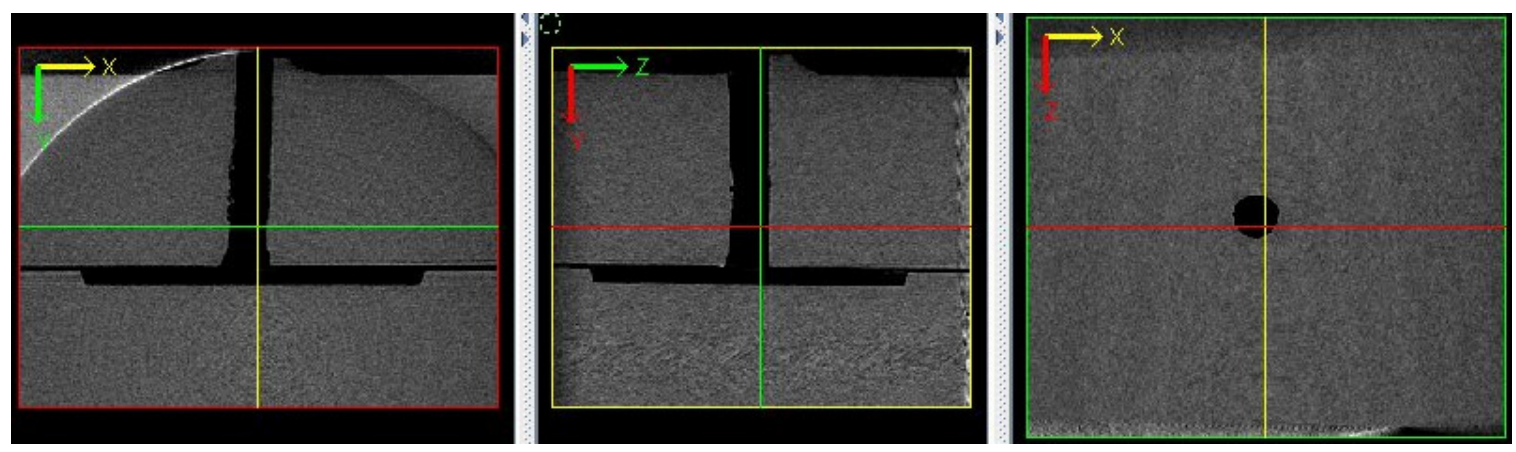

Figure 4. Orthographic views of the X-ray image at a selected location in the swirling-flow mixer packaged by screw bolting alone.
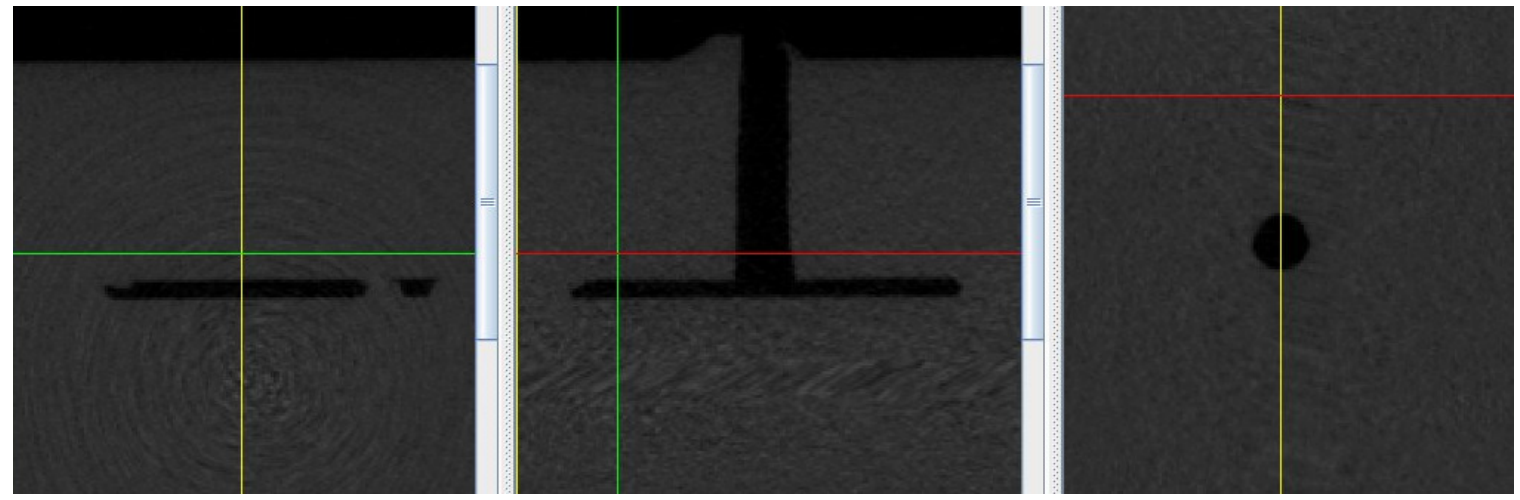

Figure 5. Orthographic views of the X-ray image at a selected location in the swirling-flow mixer packaged by tape bonding and screw bolting.

The X-ray images of the swirling-flow mixer fabricated by screw bolting are shown in figure 4 with a spatial resolution of about $20 \mu \mathrm{m}$ per pixel. As can be seen in these images, the machined mixing chamber surfaces are very smooth and flat. The thickness of the mixing chamber is also fairly uniform. The hump at the mixer outlet in the left and central panels is the dried Rhodamine ink (the pink powder in figures 1 and 2). It has a density close to that of acrylics. A small gap - about $40 \mu \mathrm{m}$ in width - between the two acrylic plates can be clearly seen in the left and central panels in figure 4. Mixing of water and Rhodamine in this mixer was examined under a bifocal microscope, and no leakage was observed. The experimental observation proved that the high surface tensions of water and Rhodamine in small acrylic channels prevented leakage through the small gap between the two acrylic plates. The X-ray images however show that this packaging is not completely airtight, thus is not suitable for gas mixing.

The images of the swirling-flow mixer fabricated by tape bonding and bolted together with two screws are shown in figure 5. A pixel size of about $40 \mu \mathrm{m}$ was used in this scanning to allow a larger volume to be scanned by the micro CT system. It was found from these X-ray images that 
the tape we used had a density very close to that of acrylics. As a result the double-sided tape and the two acrylic plates are almost indistinguishable in these images. The adhesive coatings on the two sides of the plastic tape were compressed and deformed when the two acrylic plates were pressed against each other by the screws, resulting in good sealing of the mixing chamber and flow channels and intimate contact between the tape and the acrylic plates.

It is not expected the double-sided tape can hold the two acrylic plates together when the mixer is subjected to a large pressure difference. The screws in this packaging technique therefore serve two purposes: to secure together the two acrylic plates and to provide a compression force to the flexible tape for good sealing. The imperfect matching of the plastic tape and the mixer contour can be seen in a few places such as the top left corner of the mixing chamber in the left panel in figure 5. The tape protruded into the mixing chamber is a bit blurry in this X-ray image because the tape thickness $(25 \mu \mathrm{m})$ is only half of the pixel size $(40 \mu \mathrm{m})$. Scanning with smaller pixels (which will reduce the size of the scanned volume) is needed for more accurate inspections of the alignment and matching of the layered structures when tape bonding is used for packaging.

\section{CONCLUSION}

Different packaging techniques were tested to assemble micro mixers fabricated from acrylic sheets, and the interiors of the mixers were inspected and characterized using a custom-designed micro CT system. The image acquisition and processing hardware and software of the X-ray scanner allowed convenient and thorough 3D inspection of every portion of the mixer. Plasma bonding did not work well on acrylic plates. The mixer assembled by screw bolting was not perfectly airtight, thus relying on surface tensions to prevent leakage through the small gap between the acrylic plates. X-ray images of adhesive bonding showed good contrast between the acrylics and the glue. Tape bonding provided good sealing, but densities of the tape and acrylics are very close, making it difficult to distinguish these two plastic materials in X-ray images.

\section{ACKNOWLEDGMENTS}

This work was sponsored by the Chinese National Science Council through contract number 50576014. The authors also thank Dr. Chen-Luh Lin and Dr. Bruce Gale for their technical support.

\section{REFERENCES}

[1] Stojanovic, J. N., 2006 "Structural and microstructural characteristics of B-doped PbTe semiconductor," Journal of Minerals and Materials Characterization and Engineering 5-2, pp. 143-153.

[2] Shi, S., Hwang, J. Y., 2005 "Miniaturize chemical plant with microwave heating technology." Journal of Minerals and Materials Characterization and Engineering 4-1, pp. 61-66. 
[3] Neuser, E., Suppes, A., 2007 "NanoCT visualizing internal 3D structures with submicrometer resolution," DIR 2007 - Internal Symposium on Digital Industrial Radiology and computed Tomography (June 25 - 27, 2007, Lyon, France), 18 pages.

[4] Brunke, O., Neuber, D., Lehmann, D. K., 2007 "NanoCT: Visualizing of internal 3Dstructures with submicrometer resolution," Materials Research Society Symposium Proceedings 990 Materials, Processes, Integration and Reliability in Advanced Interconnects for Micro- and Nanoelectronics, pp. 325-331.

[5] Greer, J., Sundberg, S. O., Wittwer, C. T., Gale, B. K., 2007 "Comparison of glass etching to xurography prototyping of microfluidic channels for DNA melting analysis," J. Micromech Microeng. 17, pp. 2407-2413.

[6] Qi, S., Liu, X., Ford, S., Barrows, J., Thomas, G., Kelly, K., McCandless, A., Lian, K., Goettert, J., Soper, S. A., 2002 "Microfluidic devices fabricated in poly(methyl methacrylate) using hot-embossing with integrated sampling capillary and fiber optics for fluorescence detection," Lab on a Chip 2-2, pp. 88-95.

[7] Wang, J., Chen, G., Muck, A., Collins, G. E. 2003 "Electrophoretic microchip with dualopposite injection for simultaneous measurements of anions and cations," Electrophoresis 24(21), pp. 3728-3734.

[8] Dang, F., Tabata, O., Kurokawa, M., Ewis, A. A., Zhang, L., Yamaoka, Y., Shinohara, S., Shinohara, Y., Ishikawa, M., Baba, Y., 2005 "High-performance genetic analysis on microfabricated capillary array electrophoresis plastic chips fabricated by injection molding," Anal. Chem. 77-7, pp. 2140-2146.

[9] Kelly, R. T., Pan, T., Woolley, A. T., 2005 "Phase-changing sacrificial materials for solvent bonding of high-performance polymeric capillary electrophoresis microchips," Anal. Chem. 7711, pp. 3536-3541.

[10] Horn, G., Mackin, T., Lesniak, J., Boyce, B., 2004 “A new approach for detecting defects in bonded MEMS devices," Experimental Techniques 28-5, pp. 19-22.

[11] Sasov, A., 2001 "Micro-CT for nondestructive 3D reconstruction of MEMS and sensors," Proceedings of the SPIE - The International Society for Optical Engineering 4407, pp. 388-394. [12] Lin, C. L., Miller, J. D., 2001 "A new cone-beam X-ray microtomography facility for 3D analysis of multiphase materials," 2nd World Congress on Industrial Process Tomography (Hanover, Germany, 29 ${ }^{\text {th }}-31^{\text {st }}$ August, 2001), pp. $98-109$. 